\title{
Valores genéticos para as produções de leite do dia do controle e da lactação na raça Holandesa com diferentes modelos estatísticos ${ }^{1}$
}

\section{Cláudio Manoel Rodrigues de Melo ${ }^{2}$, Irineu Umberto Packer ${ }^{3}$, Claudio Napolis Costa ${ }^{4}$, Paulo Fernando Machado ${ }^{3}$, Mateus Patrício ${ }^{5}$}

${ }^{1}$ Pesquisa financiada pela Fundação de Amparo à Pesquisa do Estado de São Paulo (FAPESP), processo 99/03838-6 e pelo Prodetab/ Embrapa, integrante do Projeto 054-02/99.

2 UFSC. Departamento de Aqüicultura - AQI, CEP: 88040-900, Florianópolis/SC, Brasil.

${ }^{3}$ Departamento de Zootecnia - USP/ESALQ, CEP: 13418-900, Piracicaba/SP, Brasil.

${ }^{4}$ Embrapa Gado de Leite, CEP: 36038-330, Juiz de Fora/MG, Brasil. Bolsista do CNPq.

${ }^{5}$ Estagiário do Laboratório de Biotecnologia Animal - Departamento de Zootecnia - USP/ESALQ.

RESUMO - Foram utilizados 263.390 registros de produção de leite do dia do controle (PDC) de 32.448 primeiras lactações de vacas da raça Holandesa paridas no período de 1991 a 2001 para predizer valores genéticos usando diferentes modelos estatísticos e a metodologia REML. Valores genéticos estimados utilizando-se três modelos de regressão aleatória (MRA) foram comparados àqueles estimados utilizando-se um modelo de repetibilidade (MRF) e um modelo para produção até 305 dias (P305). Nos modelos de regressão aleatória, duas curvas foram utilizadas para descrever a trajetória da lactação: a polinomial logarítmica (AS) e a exponencial (W), sob duas formas: padrão e com uma modificação para reduzir a amplitude das covariáveis e contornar problemas de convergência $\left(\mathrm{W}^{*}\right)$. As covariâncias requeridas foram estimadas em análises prévias utilizando-se as mesmas funções de covariância e mesmos modelos. Os desvios-padrão (DP) das estimativas de valores genéticos (EVG) para touros foram similares para os MRA com as curvas AS, W* e MRF. Os desvios-padrão das estimativas de valores genéticos para vacas e touros foram maiores nos modelos para ajuste das PDC que naquele para ajuste da P305. A magnitude das diferenças dos desviospadrão entre modelos dependeu do número de controle, no caso de vacas, e do número de filhas, no caso de touros. Os desviospadrão para o modelo $\mathrm{W}$ foram maiores, contudo, problemas de convergência ocorreram na fase de estimação dos componentes de covariância, o que exige cautela na interpretação dos resultados deste modelo. As correlações das estimativas de valores genéticos de touros preditas com os modelos testados aumentaram com o aumento no número de progênies e variaram de 0,66 (P305-W) a 0,92 (P305-AS, P305-W*). A tendência genética foi maior para os MRA e menor para MRF em comparação a P305. Os MRA possibilitam mais informações sobre as EVG que MRF por possibilitarem avaliação genética para persistência da produção de leite na lactação. Os resultados indicam o MRA com a curva de AS como o melhor modelo de ajuste das PDC alternativamente ao ajuste da P305 para avaliação genética dos animais da raça Holandesa no Brasil.

Palavras-chave: avaliação genética, correlações de ordem e de valores, modelos de regressão aleatória, produções do dia do controle

\section{Breeding values prediction for test day and lactation milk yields in Holstein cattle using different statistical models}

\footnotetext{
ABSTRACT - Data consisting of 263.390 test day records (PDC) of 32.448 first lactation Holstein cows were used to compare three random regression test-day models (RR), a repeatability test-day model (RM) and a 305-d lactation model (P305) for breeding value (EBV) prediction of milk yield. One RR model used the logarithmic function of Ali \& Schaeffer (AS) and the other two models used the exponential Wilmink function in its standard form (W) and a modified form (W*). The required covariance parameters were previously estimated from the same covariance functions and models. Standard deviations (SD) of EBVs for bulls were similar for AS, W* and RM. SD of EBVs for cows were larger for test-day models than for P305 and for bulls they differed by -33.64 to +321.95 from the P305 depending on progeny number. SD of EBVs for bulls and cows for the $\mathrm{W}$ were the largest ones. Correlation between EBVs of P305 and EBV s of the other models for bulls increased as progeny number increased and ranged from 0.66 (P305-W) to 0.92 (P305-AS, P305-W*). Genetic trends were largest for RR and smaller for RM than for P305. RR models provide more information than the RM and describe the shape of the lactation curve from which EBVs for persistency can be derived. The AS was the best function to fit RR test-day models and may be an alternative to replace the lactation model for genetic evaluation of milk yield in Holstein cattle in Brazil.
}

Key Words: genetic evaluation, random regression model, selection, test day model 


\section{Introdução}

A avaliação genética em bovinos de leite visa predizer o mérito genético dos reprodutores para orientar os criadores na seleção, no acasalamento e no descarte de animais em seus rebanhos. No caso de touros, as avaliações genéticas fornecem as informações sobre o mérito genético dos animais submetidos ao teste de progênie, identificando os touros geneticamente superiores para uso intensivo em programas de inseminação artificial, essencial para a difusão do progresso genético realizado por seleção.

O uso das produções do dia do controle (PDC) tem merecido atenção na avaliação genética de bovinos de leite em substituição à produção de leite até 305 dias (P305) e sua utilização já é uma realidade em muitos países. Na avaliação para caracteres de produção do INTERBULL de novembro de 2005, 11 países (Alemanha, Bélgica, Canadá, República Tcheca, Dinamarca, Áustria, Finlândia, Itália, Holanda, Republica Sul Africana, Estônia) submeteram suas avaliações genéticas com base nas PDC (INTERBULL, 2005). As avaliações da Estônia, da República Sul Africana e da Itália (com a raça Simental) foram feitas utilizando-se modelos de repetibilidade (MRF) e a dos demais países, modelos de regressão aleatória (MRA).

Nos MRF a variação genética é assumida constante ao longo de todo o período de lactação, o que significa que a estimativa do valor genético (EVG) para a produção de 305 dias ou qualquer período da lactação é a média genética diária predita (valor genético para produção diária) multiplicada pela duração da lactação ou pelo período da lactação considerado.

Por outro lado, nos MRA o efeito genético de animal pode ser variável para cada dia em lactação (DEL) e ser modelado por uma função de regressão aleatória (Schaeffer \& Dekkers, 1994). Assim, os MRA ajustam para a variação ambiental dentro da lactação e permitem melhor descrição dos efeitos de ambiente (Lidauer \& Mäntysaari, 1999). Adicionalmente, nos MRA os valores genéticos são estimados para cada dia ao longo da lactação e, assim, podem descrever as diferenças tanto no nível de produção como na forma da curva de lactação entre os animais, o que permite avaliações genéticas para persistência da lactação (Pool, 2000).

Neste contexto, objetivou-se neste estudo comparar estimativas de valor genético obtidas usando diferentes modelos estatísticos para ajuste das PDC e subsidiar sua utilização nas avaliações genéticas da raça Holandesa no Brasil. Compararam-se os valores genéticos preditos utilizando-se modelos de repetibilidade e de regressão aleatória para ajuste da PDC e o modelo para produção até 305 dias de primeiras lactações de vacas da raça Holandesa.

\section{Material e Métodos}

Foram utilizados registros de produção de leite coletados pelo Serviço de Controle Leiteiro (SCL) da Associação Brasileira de Criadores de Bovinos da Raça Holandesa (ABCBRH) e de suas filiadas estaduais e disponibilizados pelo Arquivo Zootécnico Nacional (AZN), gerenciado pela Embrapa Gado de Leite.

Os registros foram editados considerando grupos de contemporâneas com no mínimo quatro vacas. Adicionalmente, considerou-se que as vacas deveriam ter pelo menos três controles de produção na lactação, com o primeiro realizado até 45 dias após o parto, e idade ao parto entre 18 e 48 meses. Após essas edições, foram utilizados nas análises dados de 263.390 produções de controles mensais obtidas entre 5 e 305 dias após o parto de 32.448 primeiras lactações de vacas Holandesas e 28.437 registros de produções de leite até 305 dias.

$\mathrm{O}$ arquivo com informações de genealogia dos animais para estruturação da matriz de coeficientes de parentesco (A) foi caracterizado pela inclusão de 3.737 registros de touros aos 41.069 registros de vacas. Os animais sem produção e com apenas um descendente e ambos os pais desconhecidos foram eliminados do arquivo de pedigree, totalizando 40.770 animais na matriz A.

O modelo utilizado para o ajuste da P305 foi:

$y_{i j k}=G C_{i}+$ EPOCA $_{j}+\sum_{n=1}^{2} b_{n} x_{i j k}^{n}+a_{i j k}+e_{i j k}$

em que: $y_{i j k}=\mathrm{P} 305 ; G C_{i}=$ efeito do grupo contemporâneo $i$, formado pela combinação de rebanho-ano de parto; ÉPOCA $_{\mathrm{j}}=$ época do parto $j ; b_{1}$ e $b_{2}=$ coeficientes de regressão linear e quadrático do caráter em função da idade da vaca ao parto; $x_{i j k}=$ idade da vaca ao parto, em dias; $a_{i j k}=$ efeito genético aditivo aleatório do animal $k$ pertencente ao $G G_{i}$ e à ÉPOCA; $\mathrm{e}_{\mathrm{ijk}}=$ erro aleatório associado a cada observação considerado NID com média 0 e variância $\sigma_{e}^{2}$.

Para o ajuste das PDC sob modelo de regressão fixa (MRF), utilizou-se o modelo a seguir:

$$
y_{i j k}=R A M_{i}+E_{P O C A_{j}}+\sum_{n=1}^{2} b_{n} x_{k}^{n}+\sum_{n=1}^{4} q_{n} z_{n k}+a_{k}+p e_{k}+e_{i j k}
$$

em que: $y_{i j k}=$ PDC da vaca $k$, que pariu na época $j$ e foi controlada no grupo $i ; R A M_{i}=$ efeito do $i$-ésimo grupo de contemporâneas, formado pela combinação de rebanho, 
ano e mês do controle; ÉPOCA $A_{j}=$ época do parto $j$; $b_{1}$ e $b_{2}=$ coeficientes de regressão linear e quadrático da PDC em função da idade da vaca ao parto; $x_{k}=$ idade, em dias, da vaca $k$ ao parto $; q_{1}$ a $q_{4}=$ coeficientes de regressão da PDC em função das covariáveis $z_{n k}$, que descrevem a curva da lactação, em que: $z_{1 k}=\mathrm{c} ; z_{2 k}=\mathrm{c}^{2} ; z_{3 k}=\ln (1 / c)$; $z_{4 k}=(\ln (1 / c))^{2} \mathrm{e} \mathrm{c}=\mathrm{DEL} / 305$, sendo DEL dias em lactação; $a_{k}=$ efeito genético aditivo aleatório do animal $k ; p e_{k}=$ efeito permanente da vaca $k ; e_{i j k}=$ erro aleatório associado a cada observação considerado NID com média 0 e variância $\sigma_{e}^{2}$.

Para a modelagem da curva de lactação e da estrutura de covariância entre as PDC sob MRA, foram utilizadas as seguintes funções:

a) curva logarítmica de Ali \& Schaeffer (1987), caracterizada por cinco parâmetros, representada por AS e definida por:

$a_{0}+a_{1} c+a_{2} c^{2}+a_{3}(\ln 1 / c)+a_{4}(\ln 1 / c)^{2}$

em que $c=\mathrm{DEL} / 305$.

b) curva exponencial de Wilmink (1987), caracterizada por quatro parâmetros, representada por $\mathrm{W}$ e definida por:

$a_{0}+a_{1} t+a_{2} \exp \left(-a_{3} t\right)$

em que $t=\mathrm{DEL}$;

c) e ainda por:

$a_{0}+a_{1} t / 100+a_{2} \exp \left(-a_{3} t\right)$

em que é uma variação alternativa de $\mathrm{W}$, representada por $\mathrm{W}^{*}$; no segundo termo, a divisão de $\mathrm{t}$ por 100 foi realizada com o objetivo de reduzir a amplitude das covariáveis e melhorar as propriedades numéricas do modelo (Jakobsen et al., 2002).

Na curva de Wilmink, o quarto parâmetro $\left(a_{3}\right)$ é geralmente considerado uma constante por reduzir de quatro para três o número de parâmetros a serem estimados, o que simplifica o ajuste da curva. Nesse estudo, assumiu-se o valor de $-0,05$ para o parâmetro $a_{3}$.

Assim, em sua expressão geral, o modelo de regressão aleatória utilizado pode ser representado por:

$y_{i t}=F_{i t}+\sum_{m=0}^{f-1} \beta_{m} P_{m}(t)+\sum_{m=0}^{k-1} \alpha_{i m} P_{m}(t)+\sum_{m=0}^{k-1} \gamma_{i m} P_{m}(t)+e_{i t}$

em que: $y_{i t}$ é a produção de leite da vaca tomada no dia $t$; $\mathrm{F}_{i t}$ representa os demais efeitos fixos do modelo (efeito de rebanho-ano-mês do controle, da época do parto e da idade da vaca ao parto como covariável), com termos linear e quadrático; $B_{m}$ são os coeficientes de regressão fixa para curva média da população; $\alpha_{i m}$ e $\gamma_{i m}$ são, respec- tivamente, o s coeficientes de regressão aleatória do efeito genético aditivo e permanente de ambiente para a vaca $i$; $P_{m}(t)$ é a $m$ th função básica da curva ajustada; $f$ e $k$ são indicadores do número de coeficientes dos efeitos fixos e aleatórios da curva ajustada; e $e_{i t}$ é a medida de erro associado com classes do tempo $t$;

Assim, como para a curva AS (equação 3), tem-se:

$P_{0}(t)=1, P_{1}(t)=\mathrm{c}, P_{2}(t)=c^{2}, \mathrm{P}_{3}(t)=\ln 1 / c, \mathrm{P}_{4}(t)=(\ln 1 / c)^{2}$ e $f=k=5$

Os componentes de covariância e os valores genéticos foram estimados utilizando-se o programa DxMRR (Meyer, 1998), definindo-se como critério de convergência a diferença entre o valor da função de verossimilhança de iterações consecutivas menor que $10^{-4}$.

Nos MRA a variância genética para a produção de leite de cada DEL é uma função das covariáveis da curva ou polinômio usado no ajuste das PDC (Jamrozik \& Schaeffer, 1997). Assim, é possível calcular as EVG para cada animal ao longo da lactação. Para a curva AS, as EVG para o animal podem ser representadas como: $\hat{\mathbf{a}}_{\mathrm{i}}^{\prime}=\left[\begin{array}{lllll}\hat{a}_{0 \mathrm{i}} & \hat{\mathrm{a}}_{1 \mathrm{i}} & \hat{\mathrm{a}}_{2 \mathrm{i}} & \hat{\mathrm{a}}_{3 \mathrm{i}} & \hat{\mathrm{a}}_{4 \mathrm{i}}\end{array}\right]$, em que $\hat{\mathbf{a}}_{\mathrm{i}}^{\prime}$ é o vetor transposto dos coeficientes de regressão aleatória do animal $i$. Desse modo, a EVG do animal $i$ para a produção no dia em lactação $\mathrm{k}$ ( $\left.\hat{\mathbf{a}}_{\mathrm{i}}^{\prime}\right)$ pode ser calculada por:

$a_{0}+a_{1} c+a_{2} c^{2}+a_{3}(\ln 1 / c)+a_{4}(\ln 1 / c)^{2}$, em que DEL/305.

A EVG para a produção de leite acumulada até 305 dias, representada por $P$, pode ser obtida pela soma das EVG de cada dia ao longo da lactação.

Logo,

$\mathrm{P}=\sum_{\mathrm{k}=1}^{305} \mathrm{D}_{\mathrm{k}}$

$\mathrm{P}=\sum_{\mathrm{k}=1}^{305} \hat{\mathrm{a}}_{0 \mathrm{i}}+\hat{\mathrm{a}}_{1 \mathrm{i}} \mathrm{c}_{\mathrm{k}}+\hat{\mathrm{a}}_{2 \mathrm{i}} \mathrm{c}_{\mathrm{k}}^{2}+\hat{\mathrm{a}}_{3 \mathrm{i}}\left(\ln 1 / \mathrm{c}_{\mathrm{k}}\right)+\hat{\mathrm{a}}_{4 \mathrm{i}}\left(\ln 1 / \mathrm{c}_{\mathrm{k}}\right)^{2}$,

$\mathrm{P}=\left(\sum_{\mathrm{k}=1}^{305} 1\right) \hat{\mathrm{a}}_{0 \mathrm{i}}+\left(\sum_{\mathrm{k}=1}^{305} \mathrm{c}_{\mathrm{k}}\right) \hat{\mathrm{a}}_{1 \mathrm{i}}+\left(\sum_{\mathrm{k}=1}^{305} \mathrm{c}_{\mathrm{k}}^{2}\right) \hat{\mathrm{a}}_{2 \mathrm{i}}+\left(\sum_{\mathrm{k}=1}^{305} \ln 1 / \mathrm{c}_{\mathrm{k}}\right) \hat{\mathrm{a}}_{3 \mathrm{i}}+\left(\sum_{\mathrm{k}=1}^{305}\left(\ln 1 / \mathrm{c}_{\mathrm{k}}\right)^{2}\right) \hat{\mathrm{a}}_{4 \mathrm{i}}$ $\mathrm{P}=\left[\begin{array}{lllll}305 & 153 & 102,17 & 301,22 & 581,23\end{array}\right] \hat{\mathbf{a}}_{\mathbf{i}}$.

Do mesmo modo, a EVG do animal para a produção no dia $i$ em lactação $\mathrm{k}\left(\mathrm{D}_{\mathrm{k}}\right)$ para curva $\mathrm{W}$ pode ser calculada como: $a_{0}+a_{1} t+a_{2} \exp \left(-a_{3} t\right)$, em que $\mathrm{t}=\mathrm{DEL}$, e ainda por: $a_{0}+a_{1} t / 100+a_{2} \exp \left(-a_{3} t\right)$ para a curva $\mathrm{W}^{*}$, de modo que a EVG para produção acumulada $(\mathrm{P})$ é a soma das EVG para cada dia de lactação.

Os modelos foram comparados examinando-se os desvios-padrão das EVG obtidas de cada um, as correlações de ordem e os valores entre as EVG. Examinou-se ainda a coincidência de touros ordenados por diferentes percentuais de seleção baseada nas EVG obtidas pelo 
modelo de ajuste da P305 e pelos demais modelos de ajuste das PDC. Finalmente, analisaram-se as tendências genéticas para a produção de leite estimada por regressão da média anual das EVG de touros com progênie nascidas no período de 1988 a 1998.

As tendências genéticas $(\Delta \mathrm{G})$ para as produções de leite foram estimadas pela regressão da média ponderada e não-ponderada das EVG dos touros para cada ano. Para o ano $i$, a média ponderada da EVG do touro é: $\Delta \mathrm{G}=\left(\sum_{k} n_{k i} \cdot \mathrm{EVG}\right) / n_{. i}$, em que $n_{k i}=$ número de filhas do touro nascidas no ano $i$; EVG = estimativa dos valores genéticos do touro $k$, obtida das soluções das equações do modelo ajustado; e $n_{. i}=$ número de filhas dos touros no ano $i$.

Definiram-se como base genética as EVG dos touros com filhas nascidas no ano de 1988 preditas com o modelo P305. As EVG dos touros para os demais anos e demais modelos foram expressas com desvio desta base genética.

\section{Resultados e Discussão}

Em comparação ao modelo para a P305, os modelos para as PDC (MRF, AS, W e W*) apresentaram, para touros com pelo menos uma progênie, maiores desvios-padrão (DP) das EVG para a produção de leite (Tabela 1). Para os modelos AS e $\mathrm{W}^{*}$, o desvio-padrão para as EVG foi $24 \%$ maior que o btido pelo ajuste do modelo para P305. Quando se utilizou o MRF, o desvio-padrão foi $22 \%$ maior que para o modelo P305. Neste contexto, Reents et al. (1998) constataram aumento de $14 \%$ no desvio-padrão das EVG de touros ao utilizarem MRF em substituição ao modelo para P305. Esses resultados sugerem que modelos para as PDC permitem melhor compreensão da variabilidade existente entre os animais que os modelos para a P305 (Pool \& Meuwissen, 2001). Todavia, considerando touros com mais de 50 filhas, os resultados são diferentes. O modelo para as P305 resultou em maior desvio-padrão em comparação aos modelos para as PDC, porém, as diferenças entre o modelo para P305 e os modelos MRF, AS e $W^{*}$ foram de 2 a $3 \%$, ou seja, bem inferiores às obtidas considerando touros com pelo menos uma progênie. Este resultado confirma o observado por Lidauer et al. (2003), que relataram que a vantagem dos modelos para o ajuste das PDC é mais visível nas EVG de touros com menor número de progênie, pois aqueles com grande número de progênie recebem EVG acuradas também pelo ajuste do modelo para P305.

Observou-se também maior diferença nos desviospadrão entre os modelos para as PDC nas EVG das vacas que nas dos touros (Tabela 1). Os desvios-padrão para as EVG obtidas pelos modelos MRF, AS, W ou $\mathrm{W}^{*}$ foram

Tabela 1 - Desvios-padrão $(\mathrm{kg})$ dos valores genéticos estimados para a produção de leite, por modelo ajustado, e porcentagem (entre parênteses) em relação ao modelo para ajuste da produção até 305 dias de lactação (P305)

Table 1 - Standard deviation ( $\mathrm{kg}$ ) of predicted breeding values for milk yield, by fitted model, and percentage (between brackets) with respect to the lactation model (P305)

\begin{tabular}{|c|c|c|c|c|c|c|}
\hline \multicolumn{2}{|c|}{$\begin{array}{c}\text { Touro } \\
\text { Bull }\end{array}$} & \multicolumn{5}{|c|}{$\begin{array}{l}\text { Modelo } \\
\text { Model }\end{array}$} \\
\hline $\begin{array}{l}\text { Número de filhas } \\
\text { Number of daughters }\end{array}$ & $\begin{array}{c}\text { Número de touros } \\
\text { Number of bulls }\end{array}$ & $\mathrm{P} 305^{1}$ & MRF & AS & $\mathrm{W}^{*}$ & W \\
\hline$>399$ & 15 & $540,33(100)$ & $556,86(103)$ & $548,35(101)$ & $549,41(102)$ & $600,87(111)$ \\
\hline$>299$ & 20 & $554,37(100)$ & $542,47 \quad(98)$ & $522,98 \quad(94)$ & 520,73 (94) & $716,57(128)$ \\
\hline$>99$ & 77 & $577,85(100)$ & $561,47 \quad(97)$ & $558,38 \quad(97)$ & $554,44 \quad(96)$ & $752,75(130)$ \\
\hline$>49$ & 174 & $570,84(100)$ & $559,40 \quad(98)$ & $558,00 \quad(98)$ & $556,27 \quad(97)$ & $772,59(135)$ \\
\hline$>24$ & 316 & $569,15(100)$ & $613,66(108)$ & $602,13(106)$ & $601,89(106)$ & $849,13(149)$ \\
\hline$>0$ & 2.580 & $366,81(100)$ & $445,99(122)$ & $454,07(124)$ & $455,40(124)$ & $688,76(188)$ \\
\hline \multicolumn{2}{|l|}{$\begin{array}{l}\text { Vaca } \\
\text { Cow }\end{array}$} & \multicolumn{5}{|c|}{$\begin{array}{c}\text { Modelo } \\
\text { Model }\end{array}$} \\
\hline $\begin{array}{l}\text { Número de controles } \\
\text { Number of test day }\end{array}$ & $\begin{array}{c}\text { Número de vacas } \\
\text { Number of cows }\end{array}$ & P305 & MRF & AS & $\mathrm{W}^{*}$ & $\mathrm{~W}$ \\
\hline$>9$ & 9.415 & $398,34(100)$ & $497,83(125)$ & $538,12(135)$ & $536,59(135)$ & $730,58(183)$ \\
\hline$>7$ & 23.657 & $412,13(100)$ & $509,66(124)$ & $549,75(133)$ & $550,40(135)$ & $764,68(186)$ \\
\hline$>5$ & 27.926 & $422,08(100)$ & $518,19(123)$ & $559,61(133)$ & $561,77(135)$ & $798,80(189)$ \\
\hline$>2$ & 32.448 & $437,04(100)$ & $532,84(122)$ & $569,14(130)$ & $571,22(133)$ & $869,20(199)$ \\
\hline
\end{tabular}

${ }_{1}$ P305: modelo para o ajuste da P305; MRF: modelo de regressão fixa; AS: modelo de regressão aleatória (MRA) com a curva $a_{0}+a_{1} c+a_{2} c^{2}+$ $a_{3}(\ln 1 / c)+a_{4}(\ln 1 / c)^{2}$ e homogeneidade de variância residual, em que $c=$ DEL/305; W*: MRA com a curva $a_{0}+a_{1} t / 100+a_{2} \exp \left(-a_{3} t\right) ; W^{*}:$ MRA com a curva $a_{0}+a_{1} t+a_{2} \exp \left(-a_{3} t\right)$, em que $t=$ DEL.

1 P305: lactation model for P305; FRM: repeatability model; $A S$ : random regression model (RRM) with the curve $a_{0}+a_{1} c+a_{2} c^{2}+a_{3}(\ln 1 / c)+a_{4}(\ln 1 / c)^{2}$ and homogeneity of residual variance, where $c=D E L / 305$ and $D E L=$ days of lactation; $W:$ RRM with the curve $a_{0}+a_{1} t 100+a_{2} \exp \left(-a_{3} t\right) ; W: R R M$ with the curve $a_{0}+a_{1} t+a_{2} \exp \left(-a_{3} t\right)$, where $D E L(d a y s$ in milk). 
superiores (de 22 a 99\%) ao desvio encontrado para as EVG obtidas pelo modelo para P305. Independentemente do número de controles coletados ao longo da lactação, os desvios-padrão para as EVG obtidas pelos modelos para as PDC foram sempre superiores (22 a 99; 23 a 89; 24 a 86; e 25 a $83 \%$ maiores para vacas com mais de três, seis, oito ou com dez controles mensais, respectivamente) ao obtido pelo modelo para a P305. Esse resultado corrobora o obtido por Lidauer et al. (2003), que relataram desvios-padrão semelhantes entre EVG para vacas sem nenhuma observação e vacas com pelo menos seis controles. Contudo, as diferenças entre desvios-padrão dentro de modelos e entre grupos de vacas $(>2 ;>5 ;>7$ e $>9$ controles) foram menores.

Resultados de outros estudos (Schaeffer et al., 2000; Lidauer et al., 2000; Lidauer et al., 2003) comprovaram que modelos para as PDC em substituição aos modelos para P305 têm maior impacto nas EVG de vacas que nas de touros. Essa implicação é atribuída ao fato de que modelos para as PDC permitem melhor ajuste para os efeitos ambientais de rebanho, o que melhora principalmente a confiabilidade das EVG das vacas.

Os desvios-padrão das EVG com o modelo $\mathrm{W}$ foram superiores ( 8 a $88 \%$ para touros e 36 a $99 \%$ para vacas) aos obtidos para os demais modelos, provavelmente em virtude das maiores estimativas de herdabilidade $\left(\hat{\mathrm{h}}^{2}\right)$ obtidas pelo ajuste deste modelo. Lidauer \& Mäntysaari (1999) constataram desvio-padrão maior para EVG de controles com maior $\hat{\mathrm{h}}^{2}$. Todavia, os resultados do modelo $\mathrm{W}$ diferiram muito dos obtidos pelos demais MRA e, considerando que durante as estimativas dos componentes de covariância em análises prévias este modelo apresentou problemas de convergência, deve-se interpretar os desvios-padrão deste modelo com ressalvas.

As correlações de valores entre as EVG estimadas utilizando-se os modelos para PDC e aquelas estimadas pelo modelo para P305 variaram (Tabela 2) de 0,66 (entre as EVG do modelo W e do P305) a 0,81 (entre as EVG do modelo AS e do P305), enquanto as correlações de ordem entre esses modelos variaram de 0,68 (entre as EVG do modelo W e do P305) a 0,78 (entre as EVG do modelo AS e do P305). Entretanto, entre os modelos AS e $\mathrm{W}^{*}$, ambas as correlações de valores e de ordem foram iguais a 1. Entre os modelos avaliados, as EVG do modelo $\mathrm{W}$ foram menos correlacionadas às estimativas dos demais modelos. Quando considerados apenas touros com mais de 60 filhas (valores entre parênteses), as correlações de ordem e de valores entre os modelos para as PDC e o modelo para P305 foram superiores $(0,80$ a 0,$92 ; 0,81$ a 0,92$)$ às obtidas utilizando todos os touros.

As correlações $(0,66$ a 0,81$)$ obtidas entre as EVG estimadas pelos modelos para PDC e aquelas estimadas pelo modelo para P305 foram inferiores às reportadas por Shahrbabak (1997), de 0,905 a 0,954, e inferiores aos limites $(0,87$ a 0,97) observados na literatura (Reents \& Dopp, 1996; Jamrozik et al., 1997; Reents et al., 1998; Lidauer et al., 2000; Schaeffer et al., 2000; Emmerling et al., 2002; Reinhardt et al., 2002).

Esses resultados foram obtidos, geralmente, considerando apenas os touros ativos e com número mínimo de filhas no cálculo das correlações. Aplicando-se a restriçãode que os touros deveriam ter mais de 60 filhas, as correlações obtidas $(0,81-0,92)$ foram mais próximas às reportadas na literatura, porém ainda inferiores.Nesse contexto, Lidauer et al. (2003), considerando touros com mais de 60 filhas, reportaram correlações que variaram de 0,978 a 0,985 entre EVG obtidas por modelos para as PDC e por modelos para as P305.

A classificação dos touros utilizando as EVG obtidas pelos modelos para as PDC ou pelos modelos para a P305 apresentaram diferenças (Tabela 3). Considerando uma taxa de seleção de 5\% dos touros de maior EVG, as porcentagens de coincidência dos touros, usando as EVG obtidas

Tabela 2 - Correlações de valores (acima da diagonal) e de ordem (abaixo da diagonal) entre as estimativas de valores genéticos para touros obtidas por diferentes modelos

Table 2 - Correlations of values (above diagonal) and of order (below diagonal) between breeding values of bulls predicted from different models

\begin{tabular}{|c|c|c|c|c|c|}
\hline $\begin{array}{l}\text { Modelo } \\
\text { Model }\end{array}$ & $\mathrm{P} 305^{1}$ & MRF & AS & $\mathrm{W}^{*}$ & W \\
\hline P305 & - & $0,77(0,85)^{2}$ & $0,81(0,92)$ & $0,80(0,92)$ & $0,66(0,81)$ \\
\hline MRF & $0,73(0,87)$ & - & $0,94(0,91)$ & $0,94(0,91)$ & $0,73(0,68)$ \\
\hline AS & $0,78(0,92)$ & $0,92(0,92)$ & - & $1,00(1,00)$ & $0,89(0,91)$ \\
\hline $\mathrm{W}^{*}$ & $0,77(0,92)$ & $0,93(0,93)$ & $1,00(1,00)$ & - & $0,89(0,90)$ \\
\hline W & $0,68(0,80)$ & $0,75(0,71)$ & $0,88(0,90)$ & $0,88(0,90)$ & - \\
\hline
\end{tabular}

1 P305: modelo para o ajuste da P305; MRF: modelo de regressão fixa; AS: modelo de regressão aleatória (MRA) com a curva $a_{0}+a_{1} c+a_{2} c^{2}+$ $a_{3}(\ln 1 / c)+a_{4}(\ln 1 / c)^{2}$ e homogeneidade de variância residual, em que $c=D E L / 305 ; W^{*}:$ MRA com a curva $a_{0}+a_{1} t / 100+a_{2} \exp \left(-a_{3} t\right) ; W^{\star}: M R A$ com a curva $a_{0}+a_{1} t+a_{2} \exp \left(-a_{3} t\right)$, em que $t=\mathrm{DEL}$.

2 Valores entre parênteses são correlações entre EVG de touros com mais de 60 filhas.

1 P305: lactation model for P305; FRM: repeatability model; $A S$ : random regression model (RRM) with the curve $a_{0}+a_{1} c+a_{2} c^{2}+a_{3}(\ln 1 / c)+a_{4}(\operatorname{In} 1 / c)^{2}$ and homogeneity of residual variance,

where $c=D E L / 305$ and $D E L=$ days of lactation; $W^{*}: R R M$ with the curve $a_{0}+a_{1} t 100+a_{2} \exp \left(-a_{3} t\right) ; W$ : RRM with the curve $a_{0}+a_{1} t+a_{2} \exp \left(-a_{3} t\right)$, where $D E L($ days in milk).

${ }^{2}$ Values between brackets are correlations between EBV of sires with more than 60 daughters. 
pelas PDC em relação àquelas obtidas pela $\mathrm{P} 305$, variaram de 43,39 a 63,96\%. Considerando a mesma taxa de descarte, as porcentagens variaram de 46,36 a $60 \%$ (Tabela 3 ). Considerando os 15 touros de maior EVG e com mais de 60 filhas, 4, 4, 4 e 6 touros diferentes foram observados pela classificação entre os modelos MRF, AS, $\mathrm{W}^{*}$ e W, respectivamente, e o modelo P305. Quando considerados os 50 touros com maior EVG e com mais de 60 filhas, 7, 4, 5 e 10 touros diferentes foram observados pela classificação entre esses modelos. Assim, os resultados para este grupo de touros sugerem que a avaliação de touros usando-se as PDC conduz à seleção de praticamente o mesmo grupo de touros obtido utilizando-se as P305, embora possa ocorrer reclassificação, como indicado pelas correlações apresentadas na Tabela 2 e pelas porcentagens de coincidência descritas na Tabela 3 .

A porcentagem de coincidência entre os touros selecionados segundo classificação pelas EVG realizada pelo modelo AS, em relação àquela realizada pelas estimativas obtidas pelo modelo $\mathrm{W}^{*}$, foi superior à encontrada com os demais modelos. Considerando os 15 ou os 50 touros de maior EVG, nenhum touro diferente foi observado pela classificação entre esses modelos, o que sugere que eles ajustam de forma semelhante às diferenças entre os touros.

As EVG obtidas pelos modelos para as PDC podem ser expressas como curvas de valor genético ao longo da lactação. As trajetórias das EVG obtidas pelo MRF (Figura 1) foram retas e expressam o nível médio de produção dos animais no decorrer da lactação. Contudo, as trajetórias dos valores genéticos estimados pelo modelo AS comprovam a variabilidade genética entre os grupos de touros ao longo da lactação como desvio da curva média de valores genéticos da população. Considerando os grupos de touros com 25 ou mais filhas $(>24,>49,>99,>299,>399$ e $>499)$, as curvas de EVG foram similares em forma e variaram mais em nível de produção que no progresso da produção.

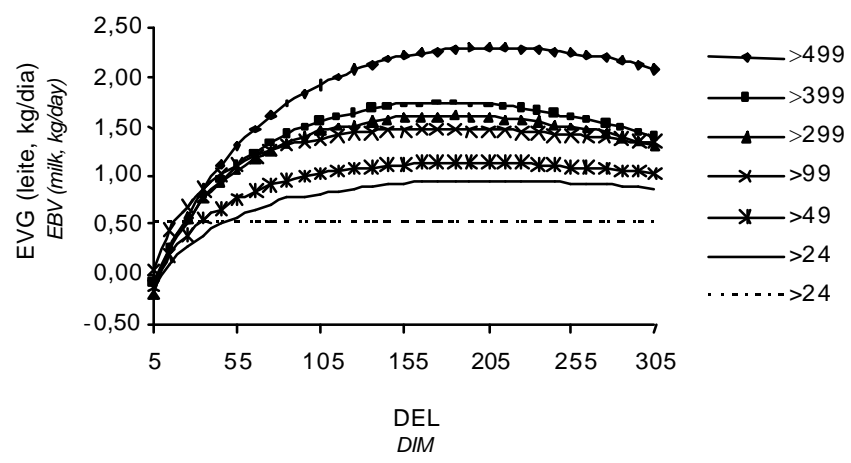

Figura 1 - Curvas de estimativa de valor genético para produção de leite de grupos de touros por número de filhas. Modelo AS (-) e modelo MRF (-----).

Figure 1 - EBVs of bulls for milk yield across lactation by group of number of progeny. AS model (-) and FRM model (-----).

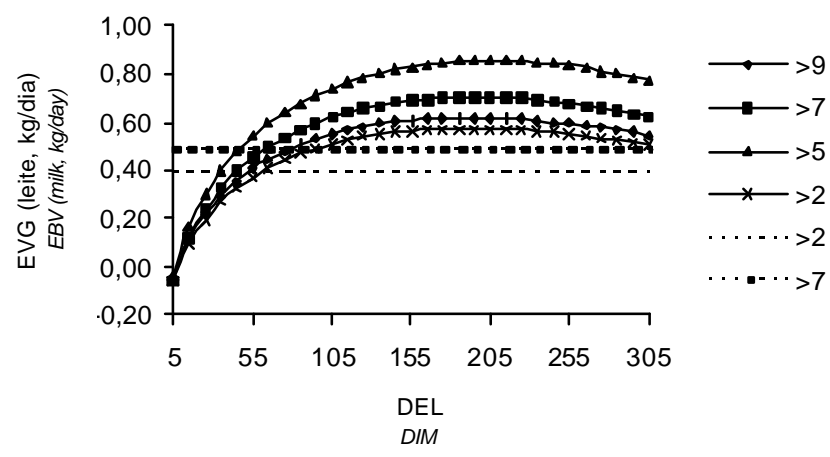

Figura 2 - Curvas de estimativa de valor genético para produção de leite de grupos de vacas por número de controles. Modelo AS (-) e modelo MRF (-----).

Figure 2 - EBVs of cows for milk yield across lactation by group of number of test days. AS model $(\longrightarrow)$ and FRM model (----).

Tabela 3 - Porcentagem de touros selecionados ou descartados, segundo a classificação pelos valores genéticos preditos, utilizando-se as produções de leite do dia do controle em relação à classificação pelos valores genéticos preditos considerando a produção de leite até 305 dias de primeiras lactações de vacas da raça Holandesa

Table 3 - Percentage of bulls selected or culled on ranking of expected breeding values for test day milk yield with respect to ranking of expected breeding values for 305 days milk yield of first lactation Holstein cows

\begin{tabular}{|c|c|c|c|c|c|c|c|c|c|c|}
\hline & \multicolumn{5}{|c|}{$\begin{array}{l}\text { Porcentagem de touros selecionados } \\
\text { Percentage of bulls selected }\end{array}$} & \multicolumn{5}{|c|}{$\begin{array}{l}\text { Porcentagem de touros descartados } \\
\text { Percentage of bulls culled }\end{array}$} \\
\hline & 5 & 10 & 20 & 30 & 50 & 5 & 10 & 20 & 30 & 50 \\
\hline $\mathrm{MRF}^{1}$ & 62,16 & 66,21 & 72,75 & 71,45 & 76,26 & 55,45 & 65,32 & 69,26 & 71,25 & 76,26 \\
\hline AS & 63,96 & 68,25 & 72,86 & 73,62 & 91,92 & 60,00 & 66,89 & 72,64 & 70,80 & 91,92 \\
\hline $\mathrm{W}^{*}$ & 63,06 & 68,25 & 72,52 & 73,78 & 81,19 & 58,64 & 67,12 & 71,85 & 69,97 & 81,19 \\
\hline W & 43,39 & 51,70 & 63,85 & 69,74 & 78,18 & 46,36 & 52,70 & 53,38 & 64,94 & 78,18 \\
\hline
\end{tabular}

${ }^{1}$ MRF: modelo de repetibilidade; AS: modelo de regressão aleatória (MRA) com a curva e homogeneidade de variância residual, em que $c=\mathrm{DEL} / 305$; $\mathrm{W}^{*}$ : MRA com a curva $a_{0}+a_{1} t / 100+a_{2} \exp \left(-a_{3} t\right) ; W^{*}:$ MRA com a curva $a_{0}+a_{1} t+a_{2} \exp \left(-a_{3} t\right)$, em que $t=\mathrm{DEL}$.

${ }^{1}$ FRM: repeatability model; $A S$ : random regression model (RRM) with the curve and homogeneity of residial variance, where $c=D E L / 305$ and $D E L=$ days of lactation; $W^{*}: R R M$ with the curve $a_{0}+a_{1} t 100+a_{2} \exp \left(-a_{3} t\right) ;$ W: RRM with the curve $a_{0}+a_{1} t+a_{2} \exp \left(-a_{3} t\right)$, where DEL (days in milk). 
Resultados semelhantes aos obtidos para diferentes grupos de touros foram encontrados para diferentes grupos de vacas (Figura 2). Nos grupos de vacas com pelo menos $3,6,8$ ou 10 controles de produção, grupos com maior número de controles apresentaram curvas de valor genético superiores às daqueles com menor número de controles em ambos os modelos, MRF (linhas pontilhadas) ou AS (linhas contínuas).

Outros pesquisadores (Pool \& Meuwissen, 2001; Pool, 2000) também obtiveram resultados semelhantes ao estimarem valores genéticos utilizando modelos semelhantes.

Curvas de valor genético para seis touros (Figura 3) foram diferentes para nível e progressão da produção. Assim, diferenças na persistência, definida como a habilidade de uma vaca em manter a produção após o pico de produção, foram evidenciadas nestas curvas. Por exemplo, as EVG para o touro 1 aumentaram continuamente até, aproximadamente, o 205을 até o fim da lactação (3050 DEL). Entretanto, para o touro 6, a curva das EVG apresentou trajetória inversa, que diminuiu do início da lactação até aproximadamente o 205 o DEL e aumentou a partir de então até o fim da lactação.

Persistência é um caráter de importância e com grande impacto da produção de leite. Em geral, produtores preferem vacas com maior persistência, pois essas vacas necessitam ingerir menos alimento para produzir a mesma quantidade de leite produzido por vacas com menor persistência de lactação; além disso, são mais facilmente alimentadas de acordo com suas exigências nutricionais, pois suas curvas de lactação são diretamente relacionadas a suas capacidades de ingestão de alimentos (Shahrbabak, 1997).

Assim, o fato de os MRA permitirem avaliar a persistência genética para produção de leite, entre outros, tem

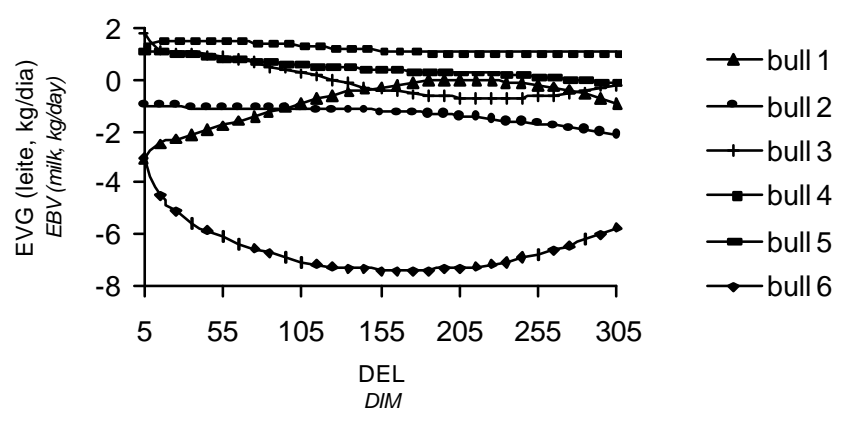

Figura 3 - Curva de estimativa de valor genético para diferentes touros. Modelo AS.

Figure 3 - EBVs of bulls for milk yield across lactation predicted by the AS model. levado vários pesquisadores (Jamrozik et al., 1997; Shahrbabak, 1997; Pool \& Meuwissen, 2001; Pool, 2000) a estudarem a viabilidade de implementar esses modelos nos sistemas de avaliação genética.

Diferentes medidas (razão da segunda parte (101-200) pela primeira parte (1-100) da lactação; porcentagem de manutenção da produção diária do pico da lactação até o fim da lactação, razão da produção na lactação pela produção diária máxima; diferença na curva de lactação entre os dias 280 e 60; entre outras (Jakobsen et al., 2002) têm sido utilizadas no cálculo da persistência. Definindo a persistência genética do animal como: $p g_{i}=\left(E V G_{i, 280}-E V G_{i, 60}\right) * 110$, em que $E V G_{i, 280}$ e $E V G_{i, 60}$ são as estimativas dos valores genéticos para o animal no DEL 280 e 60, respectivamente (Jamrozik et al., 1997), os touros 1, 2, 3, 4,5 e 6 têm persistências genéticas para produção de leite, respectivamente, de - 243,11;399,59; -5,25;361,50; 134,66 e 1.987,24. Todavia, outras medidas de persistência deveriam ser consideradas em estudos futuros.

As tendências genéticas $(\Delta \mathrm{G})$ para as produções de leite estimadas pela ponderação das EVG com os modelos P305 e MRF (Tabela 4) foram semelhantes (28,22 e 26,31 kg/ ano, respectivamente), assim como nos modelos AS e W* (48,22 e 46,2 kg/ano, respectivamente). Contudo, o modelo $\mathrm{W}$ apresentou $\Delta \mathrm{G}$ superior $(111,45 \mathrm{~kg} / \mathrm{ano})$ às dos demais modelos. A diferença entre as $\Delta \mathrm{G}$ dos diferentes modelos deveu-se, em parte, às diferenças entre as estimativas de herdabilidades obtidas pelo ajuste desses modelos. Todavia, a $\Delta \mathrm{G}$ obtida pelo modelo $\mathrm{W}$ deve ser interpretada com reservas, pois este modelo não se ajustou bem aos dados e apresentou problemas de convergência. As $\Delta \mathrm{G}$ estimadas pelas médias ponderadas foram superiores às obtidas pelas médias não-ponderadas, exceto para o MRF. No entanto, apesar de ter sido menor quando estimada pela média ponderada que pela média não-ponderada para o MRF, a $\Delta \mathrm{G}$ apresentou erro-padrão maior $(11,66)$ e $\mathrm{R}^{2}$ baixo $(0,34)$. As $\Delta \mathrm{G}$ estimadas pelas médias dos valores genéticos preditos usando o modelo $\mathrm{P} 305$ foi superior àquelas (23,88 e 32,96) estimadas por Costa (1998) utilizando média não-ponderada e ponderada, respectivamente, e considerando touros com filhas nascidas entre 1977 e 1990.

As estimativas de herdabilidade para a produção de leite pelos modelos para PDC foram maiores que a obtida para o modelo P305. As médias das herdabilidades ao longo do período de lactação para os modelos AS, W* e W foram 0,$38 ; 0,38$ e 0,54 , respectivamente. A herdabilidade para o MRF foi 0,30, enquanto, para o P305, foi 0,27, resultado que certamente contribuiu para maiores estimativas de tendência genética para esses modelos em relação ao P305. 
Tabela 4 - Estimativas do ganho genético para produção de leite (G kg/ano), respectivo erro-padrão (EP) e coeficiente de determinação $\left(\mathrm{R}^{2}\right)$ obtidos por modelos ajustados

Table 4 - Estimates of genetic gain $\left(G \mathrm{~kg}_{\text {year }}{ }^{1}\right)$ for milk yield and respective standard error (SE) and coefficient of determination ( $\left.P^{2}\right)$ by fitted model

\begin{tabular}{|c|c|c|c|c|c|c|c|c|c|c|}
\hline & \multicolumn{5}{|c|}{$\begin{array}{c}\text { EVG não-ponderada } \\
\text { Unweighted } E B V\end{array}$} & \multicolumn{5}{|c|}{$\begin{array}{l}\text { EVG ponderada } \\
\text { Weighted EBV }\end{array}$} \\
\hline & $\mathrm{P} 305^{1}$ & MRF & AS & $\mathrm{W}^{*}$ & $\mathrm{~W}$ & P305 & MRF & AS & $\mathrm{W}^{*}$ & W \\
\hline $\mathrm{G}$ & 28,22 & 26,31 & 48,03 & 46,22 & 111,45 & 39,02 & 25,17 & 52,14 & 50,30 & 114,04 \\
\hline $\operatorname{EP}(S E)$ & 2,38 & 5,78 & 2,50 & 2,48 & 8,91 & 5,97 & 11,66 & 6,04 & 5,86 & 6,67 \\
\hline $\mathrm{R}^{2}$ & 0,94 & 0,70 & 0,98 & 0,97 & 0,95 & 0,83 & 0,34 & 0,89 & 0,89 & 0,97 \\
\hline
\end{tabular}

1 P305: modelo para o ajuste da P305; MRF: modelo de regressão fixa; AS: modelo de regressão aleatória (MRA) com a curva $a_{0}+a_{1} c+a_{2} c^{2}+$ $a_{3}(\ln 1 / c)+a_{4}(\ln 1 / c)^{2}$ e homogeneidade de variância residual, em que $c=D E L / 305 ; W^{*}:$ MRA com a curva $a_{0}+a_{1} t / 100+a_{2} \exp \left(-a_{3} t\right) ; W^{*}:$ MRA com a curva $a_{0}+a_{1} t+a_{2} \exp \left(-a_{3} t\right)$, em que $t=\mathrm{DEL}$.

${ }^{1}$ P305: lactation model for P305; FRM: repeatability model; $A S$ : random regression model (RRM) with the curve $a_{0}+a_{1} c+a_{2} c^{2}+a_{3}(\operatorname{In} 1 / c)+a_{4}(\ln 1 / c)^{2}$ and homogeneity of residual variance, where $c=D E L / 305$ and $D E L=$ days of lactation; $W^{*}: R R M$ with the curve $a_{0}+a_{1} t 100+a_{2} \exp \left(-a_{3} t\right) ; W:$ RRM with the curve $a_{0}+a_{1} t+a_{2} \exp \left(-a_{3} t\right)$, where $D E L(d a y s$ in milk).

A curva $\Delta \mathrm{G}$ do modelo $\mathrm{W}$ (Figura 4$)$ foi inferior às dos demais modelos até 1993, quando superou a dos demais modelos e continuou aumentando até 1998. A diferença na curva de $\Delta \mathrm{G}$ entre o modelo $\mathrm{W}$ e os demais pode ser atribuída ao pior ajuste do modelo $\mathrm{W}$, o que ocasionou estimativas de herdabilidades altas ao longo da lactação (dados não apresentados). As médias, ponderadas ou nãoponderadas, das EVG foram semelhantes. Por exemplo, as
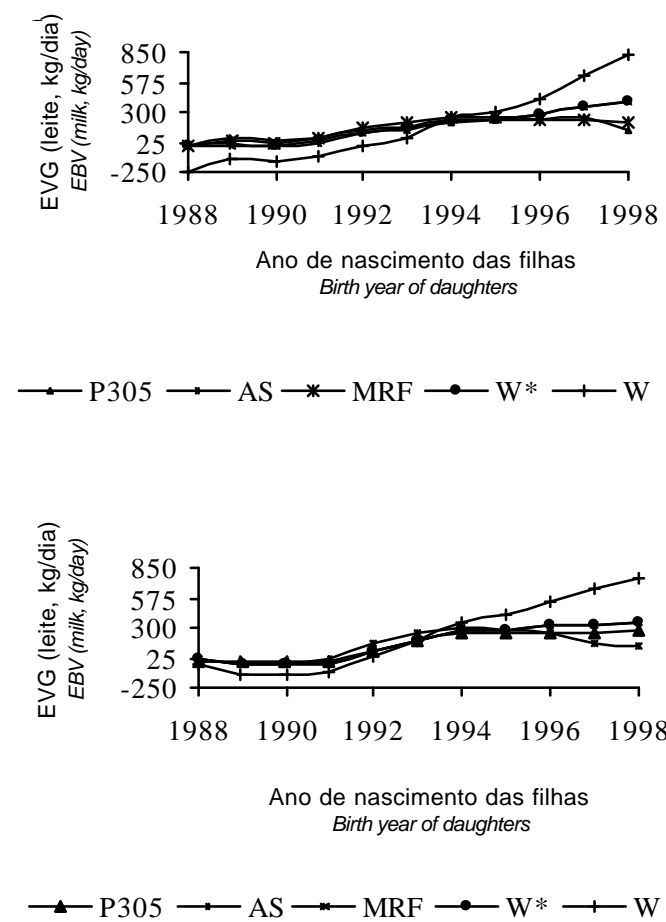

Figura 4 - Tendência genética para a produção de leite no período de 1988 a 1998 (não-ponderada à direita e ponderada à esquerda).

Figure 4 - Genetic trends for milk yield between 1988 and 1998 (unweighted mean, right side and weighted mean, left site). curvas das médias ponderadas e não-ponderadas estimadas pelos valores genéticos preditos pelos modelos $\mathrm{P} 305 \mathrm{e}$ AS foram muito semelhantes. Para esses modelos, as médias ponderadas superaram as não-ponderadas no ano de 1993, indicando que os criadores iniciaram o uso mais intensivo de touros com maior potencial genético para a produção de leite a partir desse ano. Todavia, Costa (1998) estimou médias ponderadas superiores às não-ponderadas já a partir do ano de 1987. A $\Delta \mathrm{G}$ foi pequena até o ano de 1991, mas aumentou constantemente desde então (Figuras 4 e 5). As $\Delta G$ obtidas pelas médias não-ponderadas corresponderam, respectivamente, para os modelos P305, AS, MRF, W* e W, a 0,45; 0,42; 0,77; 0,74 e 1,78\% da média $(6.256,14)$ da produção de leite das vacas Holandesas de primeira lactação. As $\Delta \mathrm{G}$ obtidas pelas médias ponderadas corresponderam a uma porcentagem ligeiramente superior $(0,62 ; 0,40 ; 0,83 ; 0,81$ e 1,83$)$, exceto para o modelo P305.

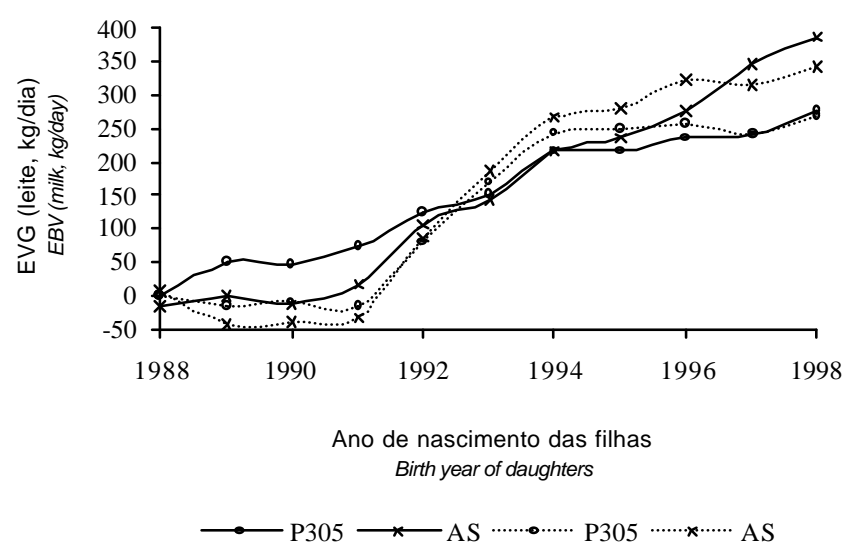

Figura 5 - Tendência genética para produção de leite no período de 1988 a 1998 (média não-ponderada-e média ponderada ------).

Figure 5 - Genetic trends for milk yield between 1988 and 1998 (unweighted mean — and weighted mean --.---). 


\section{Conclusões}

Os modelos para o ajuste das produções do dia do controle explicam melhor a variabilidade genética entre os animais que o modelo para a produção até 305 dias de lactação, principalmente entre vacas e touros com menor número de filhas. As correlações de ordem e os valores para as EVG dos touros entre o modelo P305 e os demais modelos sugerem que, para touros com maior número de progênie, seriam selecionados ou descartados praticamente os mesmos touros pelo modelo para $\mathrm{P} 305$ e pelos modelos para as PDC. As tendências genéticas foram maiores para os MRA e menores para o MRF que para o modelo $\mathrm{P} 305$, indicando que os MRA podem levar a maior progresso genético para produção de leite. Assumindo que os MRA para as PDC permitem maior ganho genético e a descrição mais detalhada dos valores genéticos dos animais ao longo da trajetória da lactação, o que permite a seleção para a persistência da lactação, esses modelos apresentam vantagens em relação ao MRF e ao ajuste da produção da lactação. Entre os modelos de regressão aleatória avaliados, o AS apresentou melhor ajuste e caracterizou-se neste estudo como o mais apropriado para o ajuste dos registros de PDC para a avaliação genética para a produção de leite na raça Holandesa no Brasil.

\section{Literatura Citada}

ALI, T.E.; SCHAEFFER, L.R. Accounting for covariances among test day milk yields in dairy cows. Canadian Journal of Animal Science, v.67, n.3, p.637-644, 1987.

COSTA, C.N. Genetic relationships for milk and fat yields between Brazilian and United States Holstein cattle populations. Ithaca: University of Cornell, 1998. 169p. Thesis (Ph.D in Philosophy) - University of Cornell, 1998.

EMMERLING, R.; LIDAUER, M.; MÄNTYSAARI, E.A. Multiple lactation random regression test-day model for Simmental and Brown Swiss in Germany and Austria. In: INTERBULL, 2002, Interlaken. Proceedings... Uppsala: International Bull Evaluation Service, 2002. p.111-117. (Bulletin, 29).

INTERBULL [2005]. Disponível em: <http://www-interbull.slu.se/ buletins/framesida-pub.htm.> Acesso em: 21/2/2005.

JAKOBSEN, J.H.; MADSEN, P.; JENSEN, J. et al. Genetic parameters for milk production and persistency for Danish Holsteins estimated in random regression models using REML. Journal of Dairy Science, v.85, n.6, p.1607-1616, 2002.

JAMROZIK, J.; SCHAEFFER, L.R. Estimates of genetic parameters for a test day model with random regressions for yield traits of first lactation Holsteins. Journal of Dairy Science, v.80, n.4, p.762-770, 1997

JAMROZIK, J.; SCHAEFFER, L.R.; DEKKERS, J.C.M. Genetic evaluation of dairy cattle using test day yields and random regression model. Journal of Dairy Science, v.80, n.6, p.12171226,1997

LIDAUER, M.; MÄNTYSAARI, E.A.; STRANDÉN, I. Comparison of test-day models for genetic evaluation of productions traits in dairy cattle. Livestock Production Science, v.79, p.7386, 2003.

LIDAUER, M.; MÄNTYSAARI, E.A; STRANDÉN, I. et al. Multiple-trait random regression test-day model for all lactations. In: INTERBULL, 2000, Bled. Proceedings... Uppsala: International Bull Evaluation Service, 2000. p.8186. (Bulletin, 25).

LIDAUER, M; MÄNTYSAARI, E.A. Multiple trait reduced rank random regression test-day model for production traits. In: INTERBULL, 1999, Zurich. Proceedings... Uppsala: International Bull Evaluation Service, 1999. p.74-80. (Bulletin 22).

MEYER, K. "DXMRR": a program to estimate covariance functions for longitudinal data by Restricted Maximum Likelihood (compact disc). In: WORLD CONGRESS OF GENETICS APPLIED LIVESTOCK PRODUCTION, 6., Armidale, 1998. Proceedings... Armidale: University of New England, 1998. (CD-ROM).

POOL, M.H. Test day models, breeding value estimation based on individual test-day records. Wageningen: University of Wageningen, 2000. 111p. Thesis (Ph.D in Philosophy) University of Wageningen.

POOL, M.H.; MEUWISSEN, T.H.E. Reduction of the number of parameters needed for a polynomial random regression test day model. Livestock Production Science, v.64, p.133-145, 2000.

POOL, M.H.; MEUWISSEN, TH.E. Effect of random regression test day models on EBVs and genetic treads in persistency. In: INTERBUlL, 2001, Rotorua. Proceedings... Uppsala: International Bull Evaluation Service, 2001. p.49-54. (Bulletin 17).

REENTS, R.; DOPP, L. Genetic evaluation for dairy production traits with a test day model for multiple lactations. In: INTERBULL, 1996, Veldhoven. Proceedings... Uppsala: International Bull Evaluation Service, 1996. p.113-117. (Bulletin 14).

REENTS, R.; DOPP, L.; SCHMUTZ, M.; REINHARDT, F. Impact of application of a test day model to dairy production traits on genetic evaluations of cows. In: INTERBULL, Rotorua, 1998. Proceedings... Uppsala: International Bull Evaluation Service, 1998, p.49-54. (Bulletin 17).

REINHARDT, F.; LIU, Z.; BÜNGER, A. et al. Impact of application of a random regression test day model to production trait genetic evaluations in dairy cows. In: INTERBULL, 2002, Interlake. Proceedings... Uppsala: International Bull Evaluation Service, 2002. p.103-107. (Bulletin 29).

SCHAEFFER, L.R.; DEKKERS, J.C.M. Random regressions in animal models for test-day production in dairy cattle (compact disc). In: WORLD CONGRESS OF GENETICS APPLIED LIVESTOCK PRODUCTION, 5., 1994, Guelph. Proceedings... Guelph: University of Guelph, 1994. v.18, p.443-446.

SCHAEFFER, L.R.; JAMROZIK, J.; KISTEMAKER, G.J. et al. Experience with a test-day model. Journal of Dairy Science, v.83, n.5, p.1135-1144, 2000.

SHAHRBABAK, M.M. Feasibility of random regression models for Iranian Holstein test day records. Guelph: University of Guelph, 1997. 138p. Thesis (Ph.D in Philosophy) - University of Guelph, 1997.

WILMINK, J.B.M. Efficiency of selection for different cumulative milk, fat, and protein yields in first lactation. Livestock Production Science, v.17, p.211-224, 1987. 